\title{
On Factors of Numbers of the Form
}

$$
\left\{x^{(2 n+1) k} \pm 1\right\} \div\left\{x^{k} \pm 1\right\} \text {. }
$$

By K. J. Sanjana, M.A.

\section{(Read 12th June 1908).}

1. In this paper the factorization of arithmetical numbers of the form $\left\{x^{2 n k+k} \pm 1\right\} \div\left\{x^{k} \pm 1\right\}$, where $x$ is a rational number such that $k x$ is a perfect square, is investigated by means of a trigonometrical transformation. The number $k$ will be taken to be prime for the present.

When $k \neq 2$, it can be at once shown that $\left(x^{k} \mp 1\right) \div(x \mp 1)$ may be a difference of two square numbers according as $k$ is of form $4 p \pm 1$. For let $\left(x^{k}-1\right) \div(x-1)$ or $x^{k-1}+x^{k-2}+x^{k-3} \ldots .+1$

$$
\begin{aligned}
\equiv\left\{x^{j^{(k-1)}}\right. & \left.+a_{1} x^{x^{(k-3)}}+a_{2} x^{(k-5)} \ldots+a_{1} x+1\right\}^{2} \\
& -k x\left\{x^{3^{(k-3)}}+b_{1} x x^{(k-5)} \ldots .+b_{1} x+1\right\}^{2} ;
\end{aligned}
$$

then $2 a_{1}-k=1$ and $2 a_{2}+a_{1}^{2}-2 b_{1} k=1$, whence $\frac{1}{4}(k+1)^{2}=1-2 a_{3}+2 b_{1} k$, so that $\frac{1}{2}(k+1)$ is odd. Iet it be $2 p+1$, then $k=4 p+1$. Similarly we can show $k=4 p-1$ or $4 p+3$ in the other case.

2. When $k$ is 2 the following proposition holds good : the number $x^{\text {tn+2 }}+1$ has four rational factors, $2 x$ being a perfect square.*

Let $2 x=y^{2}$. We have $x^{2}+1=x^{2}+2 x+1-y^{2}=(x+y+1)(x-y+1)$.

It is easily seen that

$$
\begin{aligned}
& z^{4}+1=\left(z^{2}-2 z \cos \frac{\pi}{4}+1\right)\left(z^{2}-2 z \cos \frac{3 \pi}{4}+1\right), \text { and } \\
& z^{8 n+4}+1=\left(z^{4 n+2}-2 z^{2 n+1} \cos \frac{\pi}{4}+1\right)\left(z^{4 n+2}-2 z^{2 n+1} \cos \frac{3 \pi}{4}+1\right) .
\end{aligned}
$$

- See the author's question in the Educational Times for June 1898. Numbers of the form $x^{4 n+2}+1$ have been called Bin-Aurifeuillians by Lt.-Col. Allan Cunningham, R.E., who has dealt with them in a paper "On Aurifeuillians" in the Proceedings of the Lond. Math. Soc., Vol. XXIX. (March 1898). My acknowledgments are due to Lt.-Col. Cunningham for his kindness in ellowing me to draw on this peper and on his solutions in the Reprints from the Educ. Tim. for most of my illustrative examples. 
Divide the latter equation by the former, and put $z^{2}=x$; we thus get (1)

$$
\frac{x^{4 n+2}+1}{x^{2}+1}=\frac{\left\{x^{2 n+1}-2 x^{(2 n+1)} \cos \frac{\pi}{4}+1\right\}\left\{x^{2 n+1}-2 x^{(2 n+1)} \cos \frac{3 \pi}{4}+1\right\}}{\left(x-2 x \cos \frac{\pi}{4}+1\right)\left(x-2 x+\cos \frac{3 \pi}{4}+1\right)} .
$$

Now $\cos (2 n+1) \frac{\pi}{4}=\cos \frac{\pi}{4}$ or $\cos \frac{3 \pi}{4}$, according as $n$ is of forms $4 p, 4 p+3$ or $4 p+1,4 p+2 ;$ and in these cases

$$
\cos (2 n+1) \frac{3 \pi}{4}=\cos \frac{3 \pi}{4} \text { or } \cos \frac{\pi}{4} .
$$

Therefore the right hand expression has, for every form of $n$, the following value

$$
\frac{x^{2 n+1}-2 x^{\frac{1}{2}(2 n+1)} \cos (2 n+1) \frac{\pi}{4}+1}{x-2 x^{\frac{1}{2}} \cos \frac{\pi}{4}+1} \times \frac{x^{2 n+1}-2 x^{\frac{1}{2}(2 n+1)} \cos (2 n+1) \frac{3 \pi}{4}+1}{x-2 x^{\frac{1}{2}} \cos \frac{3 \pi}{4}+1} \ldots(\alpha) .
$$

But it can be readily demonstrated that

$$
\begin{aligned}
x^{2 n-2}+\frac{\sin 2 \theta}{\sin \theta} x^{2 n-3} & +\frac{\sin 3 \theta}{\sin \theta} x^{2 n-4} \ldots+\frac{\sin n \theta}{\sin \theta} x^{n-1} \\
& +\frac{\sin (n-1) \theta}{\sin \theta} x^{n-2} \ldots+\frac{\sin 2 \theta}{\sin \theta} x+1=\frac{x^{2 n}-2 x^{n} \cos n \theta+1}{x^{2}-2 x \cos \theta+1} .
\end{aligned}
$$

Making requisite changes we obtain for (a)

$$
\begin{aligned}
& \left\{x^{2 n}+\frac{\sin \frac{2 \pi}{4}}{\sin \frac{\pi}{4}} x^{2 n-1}+\frac{\sin \frac{3 \pi}{4}}{\sin \frac{\pi}{4}} x^{2 n-1}+\frac{\sin \frac{4 \pi}{4}}{\sin \frac{\pi}{4}} x^{2 n-1} \ldots \ldots \frac{\sin \frac{3 \pi}{3}}{\sin \frac{\pi}{4}} x+\frac{\sin \frac{2 \pi}{4}}{\sin \frac{\pi}{4}} x+1\right\} \\
& \times\left\{x^{2 n}+\frac{\sin \frac{6 \pi}{4}}{\sin \frac{3 \pi}{4}} x^{2 n-1}+\frac{\sin \frac{9 \pi}{4}}{\sin \frac{\pi}{4}} x^{2 n-1} \ldots \ldots+\frac{\sin \frac{6 \pi}{4}}{\sin \frac{3 \pi}{4}} x+1\right\} \text {. }
\end{aligned}
$$


It is seen that all terms containing have $2 \frac{1}{t}$ in their coefficients, and that each of these terms has contrary signs in the two factors, and that the coefficients of the integral powers are all rational. Thus ( $\alpha$ ) is the product of two expressions of the form $P+\sqrt{2 x} Q$ and $\mathrm{P}-\sqrt{2 x} \mathrm{Q}$ or $\mathrm{P}+\mathrm{Q} y$ and $\mathrm{P}-\mathrm{Q} y$, where $\mathrm{P}$ and $\mathrm{Q}$ are rational integral functions of $x$ of degree $2 n$ and $2 n-1$ respectively. We see therefore that the left side of equation (1) has two rational factors ; and as $x^{2}+1$ has been shown to have two factors, it follows that $x^{4 n+8}+1$ has four rational factors.

3. These factors may be evaluated for given arithmetical values of $x$ and $n$. It will be found that

$$
\begin{aligned}
&\left(x^{8}+1\right) /\left(x^{2}+1\right)=\left(x^{2}+x+1\right)^{2}-y^{2}(x+1)^{2} ; \\
&\left(x^{10}+1\right) /\left(x^{2}+1\right)=\left(x^{4}+x^{3}-x^{2}+x+1\right)^{2}-y^{2}\left(x^{3}+1\right)^{2} ; \\
&\left(x^{14}+1\right) /\left(x^{2}+1\right)= \\
&\left(x^{6}+x^{3}-x^{4}-x^{3}-x^{2}+x+1\right)^{2}-y^{2}\left(x^{5}-x^{3}-x^{2}+1\right)^{2} ; \\
&\left(x^{28}+1\right) /\left(x^{2}+1\right)= \\
&\left(x^{3}+x^{7}-x^{6}-x^{3}+x^{4}-x^{3}-x^{2}+x+1\right)^{2}-y^{2}\left(x^{7}-x^{3}-x^{2}+1\right)^{2} ; \\
&\left(x^{22}+1\right) /\left(x^{2}+1\right)=\left(x^{10}+x^{9}-x^{8}-x^{7}+x^{6}+x^{5}+x^{4}-x^{3}-x^{2}+x+1\right)^{2} \\
& \quad y^{2}\left(x^{9}-x^{7}+x^{5}+x^{4}-x^{2}+1\right)^{2} ;
\end{aligned}
$$

and further similar identities can be easily obtained.

4. Examples.-(a) $242^{10}+1$.

$$
\text { Here } \quad x=242, y=\sqrt{2 x}=22 \text {; }
$$

hence

$$
\left(242^{10}+1\right) \div 58565
$$

$$
\begin{aligned}
& =\left(242^{4}+242^{3}-242^{2}+242+1\right)^{2}-22^{2}\left(242^{3}+1\right)^{2} \\
& =(3443.856263)^{2}-(311794758)^{2}:
\end{aligned}
$$

so that

$$
\mathrm{N} \equiv 242^{10}+1
$$

$$
=5 \times 13 \times 17 \times 53 \times 5 \times 626412301 \times 3755651021 .
$$

It may be shown* that $626412301=4561 \times 137341$, and $3755651021=881 \times 4262941$. The last number is prime; so that the prime factors of $\mathrm{N}$ are

$$
5^{2}, 13,17,53,881,4561,137341,4262941 .
$$

\footnotetext{
- See Reprints E. T., Vol. LXX. (Lt..Col. Cunninghsm).
} 
(b) $50^{14}+1$. Here $50^{2}+1=41 \times 61$; also $x^{6}+x^{5}-x^{4}-x^{3}-x^{2}+x+1$ $=15931122551$, and $y\left(x^{5}-x^{3}-x^{2}+1\right)=3123725010$; so that the other two factors are 19054847561 and 12807397541 . It will be found that 29 is a divisor of the given number; hence $50^{14}+1=29 \times 41 \times 61 \times 657063709 \times 12807397541$. There is no other small factor less than 151 .

(c)

$$
\mathrm{N} \equiv 9^{14}+8^{14}=8^{14}\left\{\left(\frac{9}{8}\right)^{14}+1\right\}
$$

Let $x=\frac{9}{8}$, then $y=\sqrt{2 x}=\frac{3}{2}$. Therefore

$$
\begin{aligned}
x^{14}+1 & =(x+y+1)(x-y+1)\left\{\left(x^{6}+x^{5} \ldots+1\right)^{2}-y^{2}\left(x^{5}-x^{3} \ldots 1\right)^{2}\right\} \\
& =\frac{29}{8} \cdot \frac{5}{8} \cdot \frac{480229}{8^{6}} \cdot \frac{391693}{8^{8}} .
\end{aligned}
$$

Multiplying out by $8^{14}$ we obtain

$$
9^{14}+8^{14}=5 \times 29 \times 281 \times 1709 \times 391693 .
$$

The last number is prime.

It is evident that numbers of the form $x^{4 n+2}+y^{4 n+2}$, where $2 x y$ is a perfect square, can be factorized by the method here adopted.

(d) $\mathrm{N} \equiv 18^{18}+1$. Here $18^{2}+1=5^{2} \times 13$;

$$
18^{8}+18^{7}-18^{8}-18^{5}+18^{4}-18^{3}-18^{2}+18+1=11596377655 \text {, }
$$
and

$$
6\left(18^{7}-18^{5}-18^{2}+1\right)=3661980846 ;
$$

so that the two large factors are

$$
\text { (a) } 15258501 \text { and }(\beta) \quad 7934396809 .
$$

Now $\mathrm{N}$ contains $18^{8}+1=34012225$; dividing by 325 , we see that 104653 is a factor. The prime factors of this are 229 and 457 ; and it will be found that

$$
\begin{aligned}
& (a) \div 457=33 \quad 388093, \\
& (\beta) \div 229=34648021 .
\end{aligned}
$$

It is also evident that 37 is a divisor of $\mathrm{N}$; and the last number written down is twice divisible by 37 . Thus we finally get

$$
\mathrm{N}=5^{2} \times 13 \times 37^{2} \times 229 \times 457 \times 25309 \times 33388093 .
$$

The large factor has been shown* to be prime; so that the above resolution is ultimate.

* By Mr C. E. Bickmore (Lt.-Col. Cunningham's paper, Proc. Lond. Math. Soc. XXIX.). 
(e)

$$
\begin{array}{lrl}
\quad(e) & \mathrm{N} \equiv 200^{18}+1=\left(200^{2}+1\right)\left(\mathrm{P}^{2}-y^{2} \mathrm{Q}^{2}\right) . \\
& 200^{2}+1=13.17 .181 ; \\
\text { It is seen that } & \mathrm{P}=2572735681591960201, \\
\text { that } & y \mathrm{Q}=255993599999200020 . \\
\text { and } &
\end{array}
$$$$
\text { that }
$$

and

The two large factors are therefore

and

$$
\begin{aligned}
& F_{1} \equiv 2828729281591160221 \\
& F_{2} \equiv 2316742081592760181 .
\end{aligned}
$$

Now $200^{\circ}+1$ divides $\mathrm{N}$, and has the factors 44221 and $36181(\equiv 97 \times 373)$ besides $200^{2}+1$. It is thus found that

and

$$
F_{1}=44221 \times 63968007996001 \text {, }
$$$$
F_{2}=97 \times 373 \times 64032008004001 \text {. }
$$

Writing the given number in the form

$$
(40000)^{9}+1 \equiv(37 m+3)^{9}+1 \equiv(73 m-4)^{9}+1,
$$

we get 37 and 73 as further divisors. These will be found to divide into the large factor of $F_{1}$ : so that we finally get

$$
\begin{array}{r}
200^{18}+1=13 \times 17 \times 181 \times 44221 \times 97 \times 373 \times 37 \times 73 \\
\times 23683083301 \times 64032008004001 .
\end{array}
$$

The large numbers have not been examined for factors.

(f) $32^{22}+1$. The factor $32^{2}+1=5^{2} .41$; the other two factors will be found to be $(\$ 3)$

$$
\begin{array}{ll} 
& F_{1} \equiv 1441151891495977, \\
\text { and } & F_{2} \equiv 878751140256793 .
\end{array}
$$

It will be found ${ }^{*}$ that 397 divides $F_{1}$ and 2113 divides $F_{2}$. Thus we have

$32^{22}+1=5^{2} .41 .397 .2113 .3630105520141 .415878438361$.

The large factors have not been examined.

- See Proc. Math. Soc. Lond. XXIX. (Lt.-Col. Cunningham). 
(g) $\mathrm{N} \equiv 8^{30}+1$. The factor $8^{2}+1=5.13$; the other two factors are $\quad\left(x^{2}+1\right)\left(x^{2}-1\right)^{2}\left(x^{4}+1\right)^{2}+x\left(x^{12}-x^{10}+x^{8}-x^{6}+x^{4}-x^{2}+1\right)$ $\pm y\left(x^{2}-1\right)\left(x^{4}+1\right)\left(x^{7}-1\right)$, where $x=8, y=4$. I find these to be

$$
\begin{array}{ll} 
& F_{1}=7036872740045 \\
\text { and } & F_{2}=2706490805957 .
\end{array}
$$

Writing the number in the form $512^{10}+1$, one factor is seen to be

$$
512^{2}+1=5.13 .37 .109 .
$$

Thus $\quad \mathrm{N}=5.13 .37 .73148400161 .5 .109 .12911693101$.

As $8^{10}+1$ is a factor of $\mathrm{N}$, other divisors will be found to be $1321,41,61$; and it may be shown that 181 is also a factor. Thus finally

$$
\mathrm{N}=5^{2} .13 .37 .109 .41 .61 .1321 .181 .54001 .29247661 .
$$

I have not examined the last number.

5. When $k$ is a prime greater than 2 , the following result holds good : the number $\left\{x^{(2 n+1) t} \pm 1\right\} \div\left\{x^{k} \pm 1\right\}$ has* three rational factors, $k x$ being a perfect square and the upper or lower sign being taken according as $k$ is of form $4 p-1$ or $4 p+1$. Before considering the general theorem, I shall take up the cases when $k$ is 3 and 5 .

Let $3 x=y^{2}$;

then $\quad\left(x^{3}+1\right) /(x+1)=x^{2}+2 x+1-y^{2}=(x+y+1)(x-y+1)$.

Also $z^{6}+1=\left(z^{3}-2 z \cos \frac{\pi}{6}+1\right)\left(z^{2}-2 z \cos \frac{3 \pi}{6}+1\right)\left(z^{2}-2 z \cos \frac{5 \pi}{6}+1\right)$;

changing $z$ to $x$ and transposing the middle factor,

$$
\frac{x^{3}+1}{x+1}=\left(x-2 x \cos \frac{\pi}{6}+1\right)\left(x-2 x+\cos \frac{5 \pi}{6}+1\right) \text {. }
$$

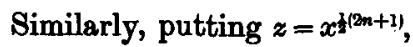

$$
\frac{x^{6 n+8}+1}{x^{2 n+1}+1}=\left\{x^{2 n+1}-2 x^{2(2 n+1)} \cos \frac{\pi}{6}+1\right\}\left\{x^{2 n+1}-2 x^{2(2 n+1)} \cos \frac{5 \pi}{6}+1\right\} \text {. }
$$

* Except when $n$ has the value $k p+\frac{1}{2}(p-1)$, in which case the general process fails. 


$$
\begin{aligned}
& \text { Hence (1) } \quad \frac{x^{6 n+3}+1}{x^{2 n+1}+1} \div \frac{x^{3}+1}{x+1} \\
& =\frac{\left\{x^{2 n+1}-2 x^{4(2 n+1)} \cos \frac{\pi}{6}+1\right\}\left\{x^{2 n+1}-2 x^{4(2 n+1)} \cos \frac{5 \pi}{6}+1\right\}}{\left(x-2 x^{3} \cos \frac{\pi}{6}+1\right)\left(x-2 x^{2} \cos \frac{5 \pi}{6}+1\right)} .
\end{aligned}
$$

Now $\cos (2 n+1) \frac{\pi}{6}=\cos \frac{\pi}{6}$ or $\cos \frac{5 \pi}{6}$, when $n$ is of form $6 p, 6 p+5$ or $6 p+2,6 p+3:$ and $\cos (2 n+1) \frac{5 \pi}{6}=\cos \frac{5 \pi}{6}$ or $\cos \frac{\pi}{6}$ in the same cases respectively. Therefore the right hand expression has, for these forms of $n$, the following value

$$
\frac{x^{2 n+1}-2 x^{2+(m+1)} \cos (2 n+1) \frac{\pi}{6}+1}{x-2 x \cos \frac{\pi}{6}+1} \times \frac{x^{2 n+1}-2 x^{(2 n+1)} \cos (2 n+1) \frac{5 \pi}{6}+1}{x-2 x+\cos \frac{5 \pi}{6}+1} .
$$

Hence as in $\S 2$, the left side of equation (1)

$$
\begin{aligned}
& =\left\{\begin{array}{c}
\sin \frac{2 \pi}{6} \\
\left.x^{2 n}+\frac{\sin \frac{3 \pi}{6}}{\sin \frac{\pi}{6}} x^{2 n-1}+\frac{\sin \frac{2 \pi}{6}}{\sin \frac{\pi}{6}} x^{2 x-1} \ldots \ldots+\frac{\sin \frac{\pi}{6}}{\sin }+1\right\} \\
\times\left\{x^{2 n}+\frac{\sin \frac{10 \pi}{6}}{\sin \frac{5 \pi}{6}} x^{2 n-1}+\frac{\sin \frac{15 \pi}{6}}{\sin \frac{5 \pi}{6}} x^{2 n-1} \ldots \ldots+\frac{\sin \frac{10 \pi}{6}}{\sin \frac{5 \pi}{6}}+1\right.
\end{array}\right\} .
\end{aligned}
$$

It is seen that the coefficients of $x^{2 n}, x^{2 n-1}, x^{2 n-2} \ldots$ are absolutely equal and rational in the two brackets; and that the coefficients of the fractional powers are equal, but opposite in sign and involve 3t throughout. Thus the expression above is the product of two factors of the form $\mathrm{P}+\sqrt{3 x} \mathrm{Q}$ and $\mathrm{P}-\sqrt{3 x} \mathrm{Q}$ or $\mathrm{P}+y \mathrm{Q}$ and $\mathrm{P}-y \mathrm{Q}$ where $P$ and $Q$ are rational integral functions of $x$ of degree $2 n$ and $2 n-1$ respectively. 
Again

$$
\begin{aligned}
\frac{x^{8 n+3}+1}{x^{3}+1} & =\left\{\frac{x^{6 n+3}+1}{x^{2 n+1}+1} \div \frac{x^{3}+1}{x+1}\right\}+\frac{x^{2 n+1}+1}{x+1} \\
& =(\mathrm{P}+y \mathrm{Q})(\mathrm{P}-y \mathrm{Q})\left(x^{2 n}-x^{2 n-1}+x^{2 n-2} \ldots-x+1\right),
\end{aligned}
$$

so that it is the product of three rational factors each of degree $2 n$; and as $x^{3}+1$ has been shown to have three factors, it follows that* $x^{b n+s}+1$ has six rational factors, when $n$ has one of the forms given above.

6. Putting $n=2,3,5$, and evaluating the coefficients above obtained, we get the following results :

$$
\begin{gathered}
\left(x^{15}+1\right) /\left(x^{3}+1\right)=\left(x^{4}-x^{3}+x^{2}-x+1\right) \times \\
\left\{\left(x^{4}+2 x^{3}+x^{2}+2 x+1\right)^{2}-y^{2}\left(x^{3}+x^{2}+x+1\right)^{2}\right\} ; \\
\left(x^{29}+1\right) /\left(x^{3}+1\right)=\left(x^{5}-x^{5}+x^{4}-x^{3}+x^{2}-x+1\right) \times \\
\left\{\left(x^{6}+2 x^{5}+x^{4}-x^{3}+x^{2}+2 x+1\right)^{2}-y^{2}\left(x^{5}+x^{4}+x+1\right)^{2}\right\} ; \\
\left(x^{33}+1\right) /\left(x^{3}+1\right)=\left(x^{10}-x^{9}+x^{8}-x^{7}+x^{6}-x^{5}+x^{4}-x^{3}+x^{2}-x+1\right)^{2} \times \\
\left\{\left(x^{10}+2 x^{9}+x^{8}-x^{7}-2 x^{6}-x^{5}-2 x^{4}-x^{3}+x^{2}+2 x+1\right)^{2}\right. \\
\left.-y^{2}\left(x^{9}+x^{8}-x^{8}-x^{5}-x^{4}-x^{3}+x+1\right)^{2}\right\} .
\end{gathered}
$$

Other similar identities can be obtained without difficulty whenever $6 n+3$ does not contain a power of 3 higher than the first.

\section{Examples:}

(a) $48^{15}+1$. Here $y=12 ; 48^{3}+1=49.37 .61$.

The other factors are $48^{4}-48^{3}+48^{2}-48+1$, and $48^{4}+2 \cdot 48^{3}+48^{2}+2 \cdot 48+1 \pm 12\left(48^{3}+48^{2}+48+1\right)$.

The given number will thus be found to be

$$
7^{2} .37 .61 .31 .134731 .5200081 .6887341 .
$$

The last two numbers have not been examined.

- See Educ. Times for August 1902. Numbers of this form have been aslled Trin-Aurifeuillians by Lt-Col. Cunninghsm in his paper " On Aurifenillians" mentioned above. As before I have derived much help from that paper in my examples. 
(b) $\mathrm{N}^{*}=972^{15}+1$. Here $x=972, y=54$;

$$
\begin{aligned}
& x^{3}+1=(x+1)(x+y+1)(x-y+1)=7.139 .919 .13 .79 ; \\
& x^{4}-x^{3}+x^{2}-x+1=891699420421=1291.690704431,
\end{aligned}
$$

where the large number is prime. It will be found that

$$
(\mathrm{P}+y \mathrm{Q})(\mathrm{P}-y \mathrm{Q})=944095306951 \times 844813520011 ;
$$

and it is easily shown that

$$
31,151,181,211,541
$$

are divisors of the given number. By actual division we obtain

$$
\begin{gathered}
\mathrm{P}+y \mathrm{Q}=31.151 .181 .211 .5281 \\
\mathrm{P}-y \mathrm{Q}=541.1561577671,
\end{gathered}
$$

and

where the large number has been shown to be prime. Thus the complete factorization of $\mathrm{N}$ is

7.139 .919 .13 .79 .1291 .31 .151 .181 .211 .541 .5281 $\times 690704431 \times 1561577671$.

(c) $12^{21}+1$. Here $12^{3}+1=13.7 .19$;

$$
12^{6}-12^{5}+\ldots-12+1=2756293 \text {; }
$$

and the remaining two factors are

$3502825 \pm 1617486$, i.e., 5120311 and 1885339 .

The first of these is divisible by 7 , and the quotient has the factors 43 and 17011 . Thus

$$
\begin{gathered}
12^{n}+1=7^{2} \cdot 13 \cdot 19: 43.17,011 \cdot 1885339.2756293 . \\
\text { (d) } 3^{111}+1 . \text { The factors of } 3^{3}+1 \text { are }+1,4,7 ; \text { and } \\
3^{30}-3^{35} \ldots-3+1=112570976472749341 .
\end{gathered}
$$

The other two factors are found to be

$$
\begin{aligned}
& \left(3^{38}+2.3^{36}+3^{34}-3^{38}-2.3^{32} \ldots-3^{17}-2.3^{16}-3^{15}+3^{14}+2.3^{13} \ldots+2.3+1\right) \\
& \pm 3\left(3^{35}+3^{34}-3^{32}-3^{31} \ldots-3^{19}-3^{16}-3^{15}+3^{13} \ldots+3+1\right), \\
& \text { i.e., } \\
& \text { and } 450283904728735897 \\
& 64326272436179833 ;
\end{aligned}
$$

223, a divisor of the number, is contained in the first of these, the quotient being 2019210335106439.

* See Reprints E. T., Vol. LXX. (Lt. -Col. Cunningham).

+1 is algebraically a factor, though it does not count numerically. 
There is no other divisor smaller than 251 .

8. When $n$ is of form $6 p+1$ or $6 p+4$, i.e., $3 q+1$, the index $6 n+3=9(2 q+1)$, and is therefore a power of 3 or a multiple of a power of 3. In this case $\cos (2 n+1) \frac{\pi}{6}$ and $\cos (2 n+1) \frac{5 \pi}{6}$ are equal to $\cos \frac{\pi}{2}$ and $\cos \frac{5 \pi}{2}$, and hence vanish; so that the right side of equation (1) cannot be put into the form

$$
\frac{x^{2 n+1}-2 x^{\frac{1}{(2 n+1)}} \cos (2 n+1) \frac{\pi}{6}+1}{x-2^{x} \cos ^{\frac{\pi}{6}}+1} \times \frac{x^{2 n+1}-2 x^{(2 n+1)} \cos \frac{5 \pi}{6}+1}{x-2 x \cos \frac{5 \pi}{6}+1},
$$

and the trigonometrical quotients of $\S 5$ cannot be obtained. We may, however, proceed algebraically thus.

$$
\text { Let } \begin{aligned}
& 6 n+3=9(2 q+1) ; \text { then }\left(x^{6 n+3}+1\right) /\left(x^{3}+1\right) \\
= & =\frac{x^{2(2 q+1)}+1}{x^{3(2 q+1)}+1} \cdot \frac{x^{3(2 q+1)}+1}{x^{3}+1}=\left\{x^{6(2 q+1)}-x^{3(2 q+1)}+1\right\} . \mathrm{E}_{1} .
\end{aligned}
$$

As shown above, $E_{1}$ is a product of three rational factors; and the bracketed expression, being $\left\{x^{3(2 q+1)}+1\right\}^{2}-y^{2}\left(x^{3 q+1}\right)^{2}$, is a difference of two squares. Hence the number $x^{6 n+3}+1$ has $8^{*}$ rational factors, including the three of $x^{3}+1$. But if $2 q+1$ is itself a multiple of $3,\left\{x^{3(29+1)}+1\right\} \div\left(x^{3}+1\right)$ has five factors, and thus the given expression has ten. In general, if $6 n+3=3 ! f$, where $f \neq 3 m$, I find the number of factors of $x^{6 n+3}+1$, given by this process, to be $2(l+2)$; but when $6 n+3=3^{l}$, the number is only $2 l+1$. But this number can be increased to at least $2 l+4$ by various artifices.

Examples.-

(a) $\left(75^{9}+1\right) \div\left(75^{3}+1\right)=\left(75^{3}+1-15.75\right)\left(75^{3}+1+15.75\right)$ and $75^{3}+1=(75+1)(75+1-15)(75+1+15)$.

Thus $75^{9}+1=76.61 .91 .420751 .423001$ $=2^{2} \cdot 19.61 .91 .127 .3313 .423001$.

The last number is prime. 
(b)

$$
\frac{48^{27}+1}{48^{3}+1}=\frac{48^{27}+1}{48^{9}+1} \cdot \frac{48^{9}+1}{48^{3}+1}
$$

$=\left(48^{\circ}+1-12.48^{4}\right)\left(48^{9}+1+12.48^{4}\right)\left(48^{3}+1-12.48\right)\left(48^{3}+1+12.48\right)$.

As $48^{3}+1=49.37 .61$, we get

$48^{27}+1=7^{2} .37 .61 .19 .5851 .110017 . F_{1} . F_{2}$ where

$F_{1}=1352605524295681, F_{2}=1352605396893697$.

(c) $12^{\text {t5 }}+1$. Let $x=12, \sqrt{3 x}=y=6$; then

$$
\begin{gathered}
x^{45}+1=\frac{x^{45}+1}{x^{15}+1} \cdot \frac{x^{15}+1}{x^{3}+1}\left(x^{3}+1\right) \\
=\left(x^{15}+1-y x^{7}\right)\left(x^{15}+1+y x^{7}\right)\left\{x^{4}-x^{3}+x^{2}-x+1\right\} \times
\end{gathered}
$$

$\left\{\left(x^{4}+2 x^{3}+x^{2}+2 x+1\right)^{2}-y^{2}\left(x^{3}+x^{2}+x+1\right)^{2}\right\}(x+1)\left\{(x+1)^{2}-y^{2}\right\}$, by $\$ 6$.

But $x^{45}+1=\left(x^{3}\right)^{15}+1$, and $3 x^{3}=y^{2} x^{2}$; hence

$$
\begin{gathered}
x^{45}+1=\left(x^{3}+1\right)\left\{\left(x^{3}+1\right)^{2}-x^{2} y^{2}\right\}\left\{x^{12}-x^{9}+x^{6}-x^{3}+1\right\} \times \\
\left\{\left(x^{12}+2 x^{9}+x^{6}+2 x^{3}+1\right)^{2}-x^{2} y^{2}\left(x^{9}+x^{6}+x^{3}+1\right)^{2}\right\},
\end{gathered}
$$

by the same formula. Now $x^{3}+1$ contains the last three factors obtained by the first process, and $x^{12}-x^{9}+x^{6}-x^{3}+1$ the preceding three. Hence the large factors $x^{15}+1 \mp y x^{7}$ are divisible by $\left(x^{3}+1\right) \mp x y$; it will be found that

$$
x^{45}+1=(x+1)(x+1+y)(x+1-y)\left(x^{4}-x^{3}+x^{2}-x+1\right) \times
$$

$\left(x^{4}+2 x^{3}+x^{2}+2 x+1-y \overline{x^{3}+x^{2}+x+1}\right)\left(x^{4}+2 x^{3}+x^{2}+2 x+1+y \overline{x^{3}+x^{2}+x+1}\right)$.

$$
\times\left(x^{3}+1+x y\right)\left(x^{3}+1-x y\right) \times
$$

$\left(x^{12}+2 x^{9}+x^{6}+2 x^{3}+1-x y \overline{x^{9}+x^{6}+x^{3}+1}\right)\left(x^{12}+2 x^{9}+x^{6}+2 x^{3}+1+x y \overline{x^{9}+x^{6}+x^{3}+1}\right)$.

We thus get $12^{45}+1$

$=13.19 .7 .19 .141 .13051 .35671 .1801 .1657 \times \mathrm{F}_{1} \cdot \mathrm{F}_{2}$; and $13051=31.421$; therefore,

$12^{45}+1=13.19 .7 .31 .421 .19141 .35671 .1801 .1657 \times$

$$
9298142 \quad 399081.8554703697721 .
$$

The last two numbers have not been tested.

(d) $3^{30}+1 \equiv \mathrm{N}$. As $\mathrm{N}=27^{33}+1$, the number has the following six factors $(\S 6)$ :

$28,19,37,198537877376983,292582128285019$, 150244883667451 .

$$
\text { As } N=\frac{3^{30}+1}{3^{33}+1}\left(3^{33}+1\right)=\left(3^{33}+1\right)\left(3^{39}-3 \cdot 3^{16}+1\right)\left(3^{33}+3 \cdot 3^{16}+1\right) \text {, }
$$

and $3^{33}+1$ has six factors $(\S 6)$, we get $\mathrm{N}=4.1 .744287$. 176419 . 25411.5559060437415361 . 5559060695695687 . 
Again 176419 and 25411 are prime; $44287=67.661$; these are the factors of 198537877376983 . Other divisors* of the given number are found to be $397,199,43 \overline{5} 7$ : and it is seen that $292582128285019=199.4357$. 337448233 , and $\quad 150244883667451=397.378450588583$.

The large quotients* have been verified to be prime; so that $N$ is completely factorised into

$$
\begin{gathered}
2^{2} .7 .19 .37 .67 .661 .25411 .176419 .199 .4357 . \\
397.337448233 .378450588583 .
\end{gathered}
$$

9. When $k$ is 5 , it may be shown that the number $x^{10 n+5}-1$ has six rational factors when $5 x$ is a square $\left(\equiv y^{2}\right)$ and the index does not contain a power of 5 higher than the first. The method of proof is not simple, and the general result cannot be easily exhibited.

As before

$$
\begin{gathered}
\frac{z^{\mathrm{N}}-1}{z^{2}-1}=\left(z^{2}-2 z \cos \frac{2 \pi}{10}+1\right)\left(z^{2}-2 z \cos \frac{4 \pi}{10}+1\right) \times \\
\left(z^{2}-2 z \cos \frac{6 \pi}{10}+1\right)\left(z^{2}-2 z \cos \frac{8 \pi}{10}+1\right) ;
\end{gathered}
$$

change $z^{2}$ to $x$ and $x^{2 n+1}$ respectively and divide: we thus obtain

$$
\text { (1) } \frac{x^{10 n+5}-1}{x^{2 n+1}-1} \div \frac{x^{5}-1}{x-1}=\frac{\Pi\left(x^{2 n+1}-2 x^{1(2 n+1)} \cos \frac{\pi}{5}+1\right)}{\Pi\left(x-2 x+\cos \frac{\pi}{5}+1\right)} \text {, }
$$

where the product $\Pi$ contains four factors involving the cosines of $\frac{\pi}{5}, \frac{2 \pi}{5}, \frac{3 \pi}{5}, \frac{4 \pi}{5}$. Also $\frac{x^{5}-1}{x-1}$

$$
\begin{aligned}
& =\left(x-2 x^{\mathrm{t}} \cos \frac{\pi}{5}+1\right)\left(x-2 x \cos \frac{2 \pi}{5}+1\right) \times\left(x-2 x \cos \frac{3 \pi}{5}+1\right)\left(x-2 x \cos \frac{4 \pi}{5}+1\right) \\
& =\left(x^{2}-\sqrt{5} x^{3}+3 x-\sqrt{5} x+1\right) \times\left(x^{2}+\sqrt{5} x^{3}+3 x+\sqrt{5} x+1\right. \\
& =\left(x^{2}+3 x+1\right)^{2}-5 x(x+1)^{2}=\left\{x^{2}+3 x+1-y(x+1)\right\}\left\{x^{2}+3 x+1+y(x+1)\right\} .
\end{aligned}
$$

* See Proc. Lond. Math. Soc., XXIX. (Lt.-Col. Cunningham.) 
It will be found that $\cos (2 n+1) \frac{\pi}{5}=\cos \frac{\pi}{5}$ or $\cos \frac{3 \pi}{5}$ according as $n$ is of forms $5 p, 5 p+4$ or $5 p+1,5 p+3$;

and

$$
\cos (2 n+1) \frac{3 \pi}{5}=\cos \frac{3 \pi}{5} \text { or } \cos \frac{\pi}{5}
$$

under the same circumstances. Similarly $\cos (2 n+1) \frac{2 \pi}{5}=\cos \frac{2 \pi}{5}$ or $\cos \frac{4 \pi}{5}$ and $\cos (2 n+1) \frac{4 \pi}{5}=\cos \frac{4 \pi}{5}$ or $\cos \frac{2 \pi}{5}$ in the same cases respectively. Hence the right hand side of (1) has, for these forms of $n$, the following value

$$
\frac{\Pi\left\{x^{2 n+1}-2 x^{\frac{1}{2}(2 n+1)} \cos (2 n+1) \frac{\pi}{5}+1\right\}}{\Pi\left\{x^{2}-2 x^{\frac{1}{2}} \cos \frac{\pi}{5}+1\right\}}
$$

that is, it is the product of

$$
f\left(\frac{\pi}{5}\right)=x^{2 n}+\frac{\sin \frac{2 \pi}{5}}{\sin \frac{\pi}{5}} x^{2 n-1}+\frac{\sin \frac{3 \pi}{5}}{\sin \frac{\pi}{5}} x^{2 n-1} \ldots+\frac{\sin \frac{3 \pi}{5}}{\sin \frac{\pi}{5}} x+\frac{\sin \frac{2 \pi}{5}}{\sin \frac{\pi}{5}} x+1
$$

and three similar series $f\left(\frac{2 \pi}{5}\right), f\left(\frac{3 \pi}{5}\right), f\left(\frac{4 \pi}{5}\right)$.

The product of the series

$$
f\left(\frac{\pi}{5}\right), f\left(\frac{2 \pi}{5}\right)
$$

is found to be

$$
x^{4 n}+\sqrt{5} \cdot x^{4 n-1}+2 \cdot x^{4 n-1}+0-2 \cdot x^{4 n-2}-\sqrt{5} \cdot x^{4 n-1} \ldots+1 ;
$$

this is of the form $\mathrm{P}+\sqrt{5 x} \mathrm{Q}$, where $\mathrm{P}$ and $\mathrm{Q}$ are rational functions of $x$ of degree $4 n$ and $4 n-1$ respectively. It will be seen that the products of

$$
f\left(\frac{3 \pi}{5}\right), f\left(\frac{4 \pi}{5}\right)
$$

is the complementary expression $\mathbf{P}-\sqrt{5 x} \mathbf{Q}$. Now

$$
\begin{aligned}
\frac{x^{10 n+5}-1}{x^{5}-1} & =\left\{\frac{x^{10 n+5}-1}{x^{2 n+1}-1} \div \frac{x^{5}-1}{x-1}\right\} \times \frac{x^{2 n+1}-1}{x-1} \\
& =(\mathrm{P}+y \mathrm{Q})(\mathrm{P}-y \mathrm{Q})\left(x^{2 n}+x^{2 n-1}+\ldots+x+1\right),
\end{aligned}
$$


so that it is the product of three rational factors ; and as $x^{5}-1$ has been proved to have three factors, it follows that $x^{10 n+5}-1$ has six rational factors.

In the excepted case $n \equiv 5 p+2$, and the index is of form $5^{2}(2 p+1)$. Here $\cos (2 n+1) \frac{\pi}{5}$ is -1 ; and the right hand side of (1) cannot be put into the form (2). The trigonometrical divisions, therefore, cannot be performed; and we shall have to proceed algebraically as in $\$ 8$.

10. In the case of $x^{15}-1$, we have $n=1$; hence, by the previous section,

$$
\begin{gathered}
f\left(\frac{\pi}{5}\right) \times f\left(\frac{2 \pi}{5}\right)^{*}=\left(x^{2}+\frac{\sin 2 \alpha}{\sin \alpha} x^{3}+\frac{\sin 3 \alpha}{\sin a} x+\frac{\sin 2 \alpha}{\sin a} x^{1}+1\right) \times \\
\left(x^{2}+\frac{\sin 4 a}{\sin 2 \alpha} x^{3}+\frac{\sin 6 a}{\sin 2 \alpha} x+\frac{\sin 4 a}{\sin 2 \alpha} x^{4}+1\right),
\end{gathered}
$$

where $a=\pi / 5$. Multiplying out and simplifying we get

$$
\begin{aligned}
& x^{4}+\sqrt{5} x^{\frac{1}{2}}+2 x^{3}+\sqrt{5} x^{4}+3 x^{2}+\sqrt{5} x^{3}+2 x+\sqrt{5} x^{4}+1, \\
& \text { that is, } \quad x^{4}+2 x^{3}+3 x^{2}+2 x+1+y\left(x^{5}+x^{2}+x+1\right) ;
\end{aligned}
$$

and the product of $f\left(\frac{3 \pi}{5}\right)$ and $f\left(\frac{4 \pi}{5}\right)$ will be found to be the complementary expression. Thus we have $\left(x^{15}-1\right) /\left(x^{5}-1\right)=$ $\left(x^{2}+x+1\right)\left\{\left(x^{4}+2 x^{3}+3 x^{2}+2 x+1\right)^{2}-y^{2}\left(x^{3}+x^{2}+x+1\right)^{2}\right\}$.

Following the same method for $n=3$ and $n=4$, I get

$$
\begin{gathered}
\left(x^{35}-1\right) /\left(x^{5}-1\right)=\left(x^{6}+x^{5}+x^{4}+x^{3}+x^{2}+x+1\right) \times \\
\left\{\left(x^{12}+2 x^{11}-2 x^{10}-x^{9}+5 x^{8}+x^{7}-3 x^{8}+x^{5}+5 x^{4}-x^{3}-2 x^{2}+2 x+1\right)^{2}\right. \\
\left.-y^{2}\left(x^{11}-x^{9}+x^{8}+2 x^{7}-x^{8}-x^{5}+2 x^{4}+x^{3}-x^{2}+1\right)^{2}\right\} \\
\left(x^{45}-1\right) /\left(x^{5}-1\right)=\left(x^{8}+x^{7}+x^{6}+x^{3}+x^{4}+x^{3}+x^{2}+x+1\right) \times \\
\left\{\left(x^{16}+2 x^{15}-2 x^{14}-x^{13}-4 x^{11}+2 x^{10}+3 x^{9}-x^{8}+3 x^{7}+2 x^{6}-4 x^{5}-x^{3}-2 x^{2}\right.\right. \\
\left.+2 x+1)^{2}-y^{2}\left(x^{15}-x^{13}-x^{11}-x^{10}+2 x^{9}+2 x^{6}-x^{5}-x^{4}-x^{2}+1\right)^{2}\right\} .
\end{gathered}
$$

* It should be noticed that in the series $f$ the co-efficients recur reciprocally after the middle term. 
11. Examples. - (a) $50000^{5}-1=49999 \times$

$\left\{50000^{2}+3 \times 50000+1 \pm 500(50001)\right\}$

$=49999.2525150501 .2475149501$.

The first large number* $=151.541 .30911$, and the second ${ }^{*}$ $=11^{2} .131 .156151$.

(b) $\mathrm{N} \equiv 320^{15}-1$. The factors of $320^{5}-1$ are 319,90521 , 116201 ; i.e., $11,29,131,691,116201$; and the number $320^{2}+320+1=139.739$. The remaining two factors are found to be 11866432681 and 9236775001 ;

the first of these ${ }^{*}=31.1951 .196201$, and the second $d^{*}=61.661$. 229081.

Thus the number is completely factorized.

(c) $20^{35}-1$. It will be found that $20^{5}-1=19.11 .61 .251$; and $20^{8}+20^{5} \ldots+1=29.71 .32719$.

The two large factors are

6527898023267251 , and 2441576160715231 .

There is no other divisor less than 251 .

(d) $\mathrm{N} \equiv 45^{25}-1=\left\{\left(45^{25}-1\right) \div\left(45^{5}-1\right)\right\}\left(45^{5}-1\right)$. The number $45^{5}-1=2^{2} .11 .2851 .1471$. Let $45^{5}=x$; then $5.45^{5}=5^{6} \cdot 3^{10}$, so that $\sqrt{5 x}=5^{3} \cdot 3^{5}=30375$. Hence

$$
\begin{array}{r}
\left(x^{5}-1\right) /(x-1)=\left\{x^{2}+3 x+1+y(x+1)\right\}\left\{x^{2}+3 x+1-y(x+1)\right\} \\
=34056234511427251.34045024427772751 .
\end{array}
$$

There is no other divisor less than 200.

$$
\begin{aligned}
& \text { (e) } \mathrm{N} \equiv 5^{75}-1=\frac{5^{75}-1}{5^{15}-1} \cdot \frac{5^{15}-1}{5^{5}-1} \cdot\left(5^{5}-1\right) \\
& =\left\{5^{30}+3.5^{15}+1+5^{8}\left(5^{15}+1\right)\right\}\left\{5^{30}+3.5^{15}+1-5^{8}\left(5^{15}+1\right)\right\} \\
& \times\left(5^{2}+5+1\right)\left\{\left(5^{4}+2.5^{3}+3.5^{2}+2.5+1\right)^{2}-5^{2}\left(5^{3}+5^{2}+5+1\right)^{2}\right\} \\
& \times(5-1)\left\{5^{2}+3.5+1+5(5+1)\right\}\left\{5^{2}+3.5+1-5(5+1)\right\} \\
& =2^{2} \cdot 11.71 .31 \cdot 181.1741 \cdot \mathrm{F}_{1} \cdot \mathrm{F}_{2} . \\
& \text { Also } \mathrm{N}=\frac{5^{75}-1}{5^{25}-1} \cdot \frac{5^{25}-1}{5^{5}-1} \cdot\left(5^{5}-1\right), \text { putting } 5^{5}=x \text { and } 5^{6}=y^{2}, \\
& \quad=\left(5^{5}-1\right)\left\{\left(x^{2}+3 x+1\right)^{2}-y^{2}(x+1)^{2}\right\} \times \\
& \quad\left(x^{2}+x+1\right)\left\{\left(x^{4}+2 x^{3}+3 x^{2}+2 x+1\right)^{2}-y^{2}\left(x^{3}+x^{2}+x+1\right)^{2}\right\} \\
& =2^{2} .11 .71 .9384251 .10165751 .9768751 . \mathrm{G}_{1} \cdot \mathrm{G}_{2} .
\end{aligned}
$$

It will be found that $9768751=31.181 .1741, F_{1}=G_{1} \times 9384251$, and $F_{2}=G_{2} \times 10165751$.

*Reprinte E.T., Vol. LXX. (Lt. Col. Cunningham). 
Other small divisors of the number are seen to be 101, 151, 251. Thus $\mathrm{N}=2^{2} .11 .71 .31 .181 .1741 .9384251 .101 .251 .401$. 151.606705812 851 .99244414 459501 . The large factors have not been tested.

12. It is now easy to see that the number $x^{(2 n+3) t} \pm 1$ has six rational factors. In the first place we have

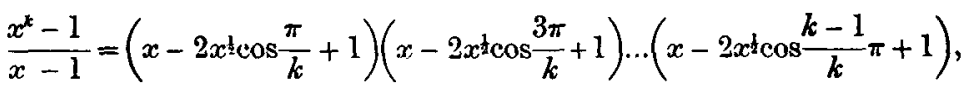
when $k=4 p+1$; and $\frac{x^{k}+1}{x+1}=\left(x-2 x \cos \frac{\pi}{2 k}+1\right)\left(x-2 x \cos \frac{3 \pi}{2 k}+1\right) \ldots\left(x-2 x+\cos \frac{2 k-1}{2 k} \pi+1\right)$ when $k=4 p+3$. In the second case the factor containing $\cos \frac{k \pi}{2 k}$ is absent from the right side, being in fact the denominator of the left; thus the number of trigonometrical factors is $k-1$ in both cases. It will be found that these can always be arranged in two groups each of $\frac{1}{2}(k-1)$ factors whose products are severally of forms $\mathbf{P}+\sqrt{k x} \mathrm{Q}, \mathrm{P}-\sqrt{k x} \mathrm{Q}$, where $\mathrm{P}$ and $\mathrm{Q}$ are rational functions of $x$ of degree $\frac{1}{2}(k-1)$ and $\frac{1}{2}(k-3)$ respectively. Thus $\left(x^{k} \mp 1\right) /(x \mp 1)$ has two rational factors. In the next place, we put $x^{(2 n+1) k} \pm 1$ in the form (A)

$$
\left\{\frac{x^{(2 n+1) k} \pm 1}{x^{2 n+1} \pm 1} \div \frac{x^{k} \pm 1}{x \pm 1}\right\} \frac{x^{2 n+1} \pm 1}{x \pm 1}\left(x^{k} \pm 1\right)
$$

As before, we can prove

$$
\begin{gathered}
\frac{x^{(2 n+1) k}+1}{x^{2 n+1}+1}=\left\{x^{2 n+1}-2 x^{2(2 n+1)} \cos \frac{\pi}{2 k}+1\right\}\left\{x^{2 n+1}-2 x^{\left.\frac{1(2 n+1)}{2} \cos \frac{3 \pi}{2 k}+1\right\}}\right. \\
\ldots\left\{x^{2 n+1}-2 x^{4(2 n+1)} \cos \frac{2 k-1}{2 k} \pi+1\right\},
\end{gathered}
$$

and a similar result for $\left\{x^{(2 n+1) k}-1\right\} /\left\{x^{2 n+1}-1\right\}$. Hence the expression in large brackets in (A) is

$$
\frac{\Pi\left\{x^{2 n+1}-2 x^{2(2 n+1)} \cos \frac{\pi}{2 k}+1\right\}}{\Pi\left\{x-2 x^{2} \cos \frac{\pi}{2 k}+1\right\}} \text { or } \frac{\Pi\left\{x^{2 n+1}-2 x^{2} \cos \frac{\pi}{k}+1\right\}}{\Pi\left\{x-2 x^{2} \cos \frac{\pi}{k}+1\right\}}
$$


according as $k$ is $4 p+3$ or $4 p+1$. The products $\Pi$ contain each an even number of factors $k-1$; and as

$$
\cos (2 n+1) \frac{\pi}{2 k}, \cos (2 n+1) \frac{3 \pi}{2 k}, \ldots \cos (2 n+1) \frac{2 k-1}{2 k} \pi
$$

have the values

$$
\cos \frac{\pi}{2 k}, \cos \frac{3 \pi}{2 k}, \ldots \cos \frac{2 k-1}{2 k} \pi
$$

in same order depending on the values of $k^{*}$, as also

$$
\cos (2 n+1) \frac{\pi}{k}, \cos (2 n+1) \frac{2 \pi}{k}, \ldots \cos (2 n+1) \frac{k-1}{k} \pi
$$

have the values $\cos \frac{\pi}{k}, \cos \frac{2 \pi}{k}, \ldots \cos \frac{k-1}{k} \pi$ in same order, it follows that the products take the form

$$
\begin{gathered}
\frac{\Pi\left\{x^{2 n+1}-2 x^{(2 n+1)} \cos (2 n+1) \frac{\pi}{2 k}+1\right\}}{\Pi\left\{x-2 x^{\frac{1}{2}} \cos \frac{\pi}{2 k}+1\right\}} \\
\text { or } \frac{\Pi\left\{x^{2 n+1}-2 x^{2(2 n+1)} \cos (2 n+1) \frac{\pi}{k}+1\right\}}{\Pi\left\{x-2 x^{3} \cos \frac{\pi}{k}+1\right\}} .
\end{gathered}
$$

Thus the expression is the product of $k-1$ series of the form

$$
\begin{gathered}
x^{2 n}+\frac{\sin \frac{2 \pi}{2 k}}{\sin \frac{\pi}{2 k}} x^{2 n-\frac{1}{2}}+\frac{\sin \frac{3 \pi}{2 k}}{\sin \frac{\pi}{2 k}} x^{2 n-1} \ldots+\frac{\sin \frac{2 \pi}{2 k}}{\sin \frac{\pi}{2 k}} x+1, \\
\text { or } x^{2 n}+\frac{\sin \frac{2 \pi}{k}}{\sin \frac{\pi}{k}} x^{2 n-1}+\frac{\sin \frac{3 \pi}{k}}{\sin \frac{\pi}{k}} x^{2 n-1} \ldots+\frac{\sin \frac{2 \pi}{k}}{\sin \frac{\pi}{k}} x+1,
\end{gathered}
$$

- The value of $\cos (2 n+1) \frac{k \pi}{2 k}$ is zero, and the factor corresponding to this function does not occur in the product. 
in the two cases. Hence, always the expression referred to is the product of $k-1$ such trigonometrical series. It will be found* that these can always be arranged in two groups, each of $\frac{1}{2}(k-1)$ series, such that the products in the groups are of forms $\mathbf{P}^{\prime}+\sqrt{k x} \mathrm{Q}^{\prime}, \mathrm{P}^{\prime}-\sqrt{k x} \mathrm{Q}^{\prime}$, where $\mathbf{P}^{\prime}$ and $\mathbf{Q}^{\prime}$ are rational functions of $x$ of degree $n(k-1)$ and $n(k-1)-1$ respectively. Thus

$$
x^{(2 n+1) k} \pm 1=\left\{P^{\prime}+\sqrt{k x} Q^{\prime}\right\}\left\{P^{\prime}-\sqrt{k x} Q^{\prime}\right\}\left(x^{2 n} \mp x^{2 n-1} \ldots+1\right)\left(x^{k} \pm 1\right) ;
$$

and as the last factor has been shown to have three rational factors, it follows that the given number has six such factors.

13. I conclude by giving a few formulæ for the values $7,11,13$ of $k$.

The expression $x^{14}+1$ is the product of seven factors of the form $x^{2}-2 x \cos \frac{k \pi}{14}+1$; of these the central factor is $x^{2}+1$. Hence $\left(x^{14}+1\right) /\left(x^{2}+1\right)$ is the product of six such factors; and changing $x^{2}$ to $x$ we get

$$
\begin{gathered}
\left(x^{7}+1\right) /(x+1)=\left(x-2 \sqrt{x} \cos \frac{\pi}{14}+1\right)\left(x-2 \sqrt{x} \cos \frac{3 \pi}{14}+1\right) \\
\times\left(x-2 \sqrt{x} \cos \frac{5 \pi}{14}+1\right)\left(x-2 \sqrt{x} \cos \frac{9 \pi}{14}+1\right)\left(x-2 \sqrt{x} \cos \frac{11 \pi}{14}+1\right) \\
\left(x-2 \sqrt{x} \cos \frac{13 \pi}{14}+1\right)
\end{gathered}
$$

It will be found that the factors containing $\frac{\pi}{14}, \frac{3 \pi}{14}, \frac{9 \pi}{14}$ give rise to a product of the form $\mathrm{P}+\sqrt{7 x} \mathrm{Q}$; the others to the complementary expression. Hence

$$
x^{7}+1=(x+1)\left\{\left(x^{3}+3 x^{2}+3 x+1\right)^{2}-7 x\left(x^{2}+x+1\right)^{2}\right\} \text {. }
$$

Also, changing $x^{2}$ to $x^{3}$ we get

$$
\left(x^{21}+1\right) /\left(x^{3}+1\right)=\Pi\left(x^{3}-2 x \cos \frac{\pi}{14}+1\right),
$$

where there are six factors. Thus

$$
\frac{x^{21}+1}{x^{3}+1} \div \frac{x^{7}+1}{x+1}=\frac{\Pi\left(x^{3}-2 x \cos \frac{\pi}{14}+1\right)}{\Pi\left(x-2 x^{2} \cos \frac{\pi}{14}+1\right)}
$$

* I have no right proof to offer of this statement. 
As $\quad \cos 3 \cdot \frac{\pi}{14}=\cos \frac{3 \pi}{14}, \cos 3 \cdot \frac{3 \pi}{14}=\cos \frac{9 \pi}{14}, \cos 3 \cdot \frac{9 \pi}{14}=\cos \frac{\pi}{14}$,

it is seen that the three factors above involving

$$
\frac{\pi}{14}, \frac{3 \pi}{14}, \frac{9 \pi}{14}
$$

are divisible by the factors below involving

$$
\frac{9 \pi}{14}, \frac{\pi}{14}, \frac{3 \pi}{14}
$$

respectively : similar remarks apply to the remaining three factors. Hence the above quantity is the product of the following two groups of series

$$
f\left(\frac{\pi}{14}\right), f\left(\frac{3 \pi}{14}\right), f\left(\frac{9 \pi}{14}\right) \text { and } f\left(\frac{5 \pi}{14}\right), f\left(\frac{11 \pi}{14}\right), f\left(\frac{13 \pi}{14}\right) .
$$

The former product will be found to be

$$
x^{6}+4 x^{5}-x^{4}-7 x^{3}-x^{2}+4 x+1+\sqrt{7 x}\left(x^{5}+x^{4}-2 x^{3}-2 x^{2}+x+1\right) ;
$$

the latter to be the complementary expression. Hence finally

$$
\begin{gathered}
x^{21}+1=\left(x^{7}+1\right)\left(x^{2}-x+1\right) \times \\
\left\{\left(x^{6}+4 x^{5}-x^{4}-7 x^{3}-x^{2}+4 x+1\right)^{2}-7 x\left(x^{5}+x^{4}-2 x^{8}-2 x^{2}+x+1\right)^{2}\right\} .
\end{gathered}
$$

Similarly changing $x^{2}$ to $x^{5}$ in the original identity, I find that

$$
\begin{gathered}
x^{35}+1=\left(x^{7}+1\right)\left(x^{4}-x^{3}+x^{2}-x+1\right) \times \\
\left\{\left(x^{12}+4 x^{11}+6 x^{10}+11 x^{9}+15 x^{8}+17 x^{7}+19 x^{6}+17 x^{5}+15 x^{4}+11 x^{3}+6 x^{2}+4 x+1\right)^{2}\right. \\
\left.-7 x\left(x^{11}+2 x^{10}+3 x^{9}+5 x^{8}+6 x^{7}+7 x^{6}+7 x^{5}+6 x^{4}+5 x^{3}+3 x^{2}+2 x+1\right)^{2}\right\} \\
\text { When } k=11, \quad\left(x^{11}+1\right) /(x+1)=\Pi\left(x^{k}-2 x^{1} \cos \frac{k \pi}{22}+1\right),
\end{gathered}
$$

where $\Pi$ includes 10 factors. The product of five of these involving the cosines of

$$
\frac{\pi}{22}, \frac{5 \pi}{22}, \frac{7 \pi}{22}, \frac{9 \pi}{22} \text { and } \frac{19 \pi}{22}
$$

will be found to be

$$
x^{5}+5 x^{4}-x^{3}-x^{2}+5 x+1+\sqrt{11 x}\left(x^{4}+x^{3}-x^{2}+x+1\right) ;
$$


that of the other five is the complementary surd. When $k=13$, we have to combine the six factors involving the cosines of

$$
\frac{\pi}{13}, \frac{2 \pi}{13}, \frac{3 \pi}{13}, \frac{6 \pi}{13}, \frac{8 \pi}{13} \text { and } \frac{9 \pi}{13},
$$

as also the six remaining ones. The following result is thus obtained

$$
\begin{aligned}
x^{13}-1=(x-1)\left\{\left(x^{8}+7 x^{5}\right.\right. & \left.+15 x^{4}+19 x^{3}+15 x^{2}+7 x+1\right)^{2} \\
& \left.-13 x\left(x^{5}+3 x^{4}+5 x^{3}+5 x^{2}+3 x+1\right)^{2}\right\} .
\end{aligned}
$$

